\title{
An Observer Based Distributed Controller for Formation Flying of Satellites
}

\author{
Prathyush P Menon and Christopher Edwards
}

\begin{abstract}
In this paper, a novel nonlinear observer and controller framework is suggested for achieving formation control of a cluster of satellites. Exploiting the skew symmetry in the satellite dynamics, a novel nonlinear observer which has roots in the super-twist sliding mode observer is proposed. Estimation of the entire states and unknown bounded disturbances (and also faulty, corrupted leader control signals) in finite time is demonstrated using an elegant Lyapunov analysis. The proposed distributed controller is based on the state estimates and the relative position output information which depends on the underlying communication topology. The novelty in the synthesis of the controller is mainly in the treatment of the underlying graph topology, the interaction amongst the satellites in terms of relative sensing, and the synthesis of the controller gains using a simple polytopic representation that depends on the graph Laplacian eigenvalues. A simulation example is provided to demonstrate the efficacy of the proposed approach.
\end{abstract}

\section{INTRODUCTION}

In recent years, the deployment of multiple, cheap costeffective satellites into the earth orbit and enabling them to perform together to meet a common task in a specified pattern has become attractive. Centralised, decentralized and distributed approaches have been developed. Many researchers have investigated the problem from different (personally) favoured approaches. From the operation cost perspective, optimal control laws are preferred to account for the deviations from a desired orbit ( $J_{2}$ invariant orbit) caused by multiple external sources such as atmospheric drag, solar and lunar gravity and most significantly due to the Earth's oblateness (also known as $J_{2}$ effect). For details on these aspects, the interested reader is referred to [1]-[4].

Because the inter satellite distances are small compared to the radii of the orbits, the equations of motion governing the relative behaviors of the satellites in the formation can be represented by Hill's equations [2](or the Clohessy Wiltshire equations [5]). Many of the control law developments which have appeared for formation flying have been based on Hill's equations [6], [7]. Different leader follower paradigms, multiple topologies and control paradigms have been considered by different researchers. In particular graph theoretic approaches, a more recent advance in looking at this class of problems, and its application to formation problems are addressed in [8]-[14] and the references therein. Sliding mode principles have been utilised in [6], [7] for formation control of leader-follower configuration. A behavioural approach for an identical problem is studied in [15]. Universal adaptive gain control has been employed in [16] to obtain a less conservative and generic adaptive position tracking of formation of satellites. In [16] the underlying dynamics considered were not Hill's equations but fully nonlinear as in [17] where a novel learning scheme

Prathyush P. Menon is affiliated to the Centre for Systems, Dynamics and Control, Mathematical Research Institute, School of Engineering, Mathematics and Physical Sciences, University of Exeter, Exeter, EX4 4QF, UK. P.M.Prathyush@exeter.ac.uk

Christopher Edwards is affiliated to Department of Engineering, University of Leicester, Leicester, LE1 7RH, UK. chris.edwardsele.ac.uk has been realized for formation control. Estimation of the entire states of the system from a set of measurements for use in control law, has been looked in [18], [19]. The former is based on the covariance estimation principles, whilst the latter employs a fuzzy paradigm.

In [20] the application of graph theoretic methods to a number of different applications including satellite formation problems [14] is discussed. Ref. [11] provides a detailed look at consensus protocols for a similar problems. According to [8], [10]-[12], [14], formation flying of multiple satellites can be modeled as a spatially distributed network of vehicles which collaborate to perform a unique mission requirement. Graph theoretic methods that are discussed in detail in [20], have been exploited in [10] to address the formation flying problem from the perspective of a synchronisation of a network of Lagrangian systems. An attractive generalised framework, which also utilizes the basics of contraction theory, is proposed in [10]. In [9] linear controllers have been designed using the relative measurements together with an observer. In addition, the possibility of decentralization of the designed controllers, and possible switching of the underlying communication topologies with out affecting the stability and performance of the collective system, have also been investigated. Refs. [21], [22] discuss distributed control and decentralized estimation architectures for formation flying. In addition, [23] reports a fuel optimal control design by addressing disturbance rejection.

Sliding mode tracking control laws (decoupled in terms of in-plane and cross-track) are developed in [6] in order for the follower satellites to maintain a close formation with a leader satellite. The underlying dynamics considered for the design are the Hill equations. Nonlinear simulations are carried out in the presence of disturbances to validate the efficacy of the proposed scheme. Continuous sliding mode control schemes driven by a sliding mode disturbance observer and a formation controller using a super twisting second order sliding mode controller are proposed in [7]. In addition, an integral sliding mode controller is also proposed in [7]. All the sliding mode control methods are implemented pulse width modulation techniques for precision robust tracking.

In this paper, a leader, and a cluster of multiple satellites is considered and treated as a spatially distributed network of dynamical systems. The individual satellite dynamics are studied using the Hill equations (as many other similar studies as discussed above). A novel nonlinear observer, exploiting structures in the satellite dynamics, is proposed to robustly estimate the states and the disturbances in the follower satellites from the relative position information only (together with the leader information). Furthermore, a robust distributed output feedback control scheme is developed in an elegant way treating the network system from a polytopic perspective. 


\section{System Description AND PRoblem Definition}

A cluster of $N+1$ satellites, consisting of a leader satellite and $N$ follower satellites, which are in nearby orbits, is considered. The following assumptions are imposed. The leader satellite is on a circular Keplerian orbit. The follower satellites can estimate the relative distance between all the nearby satellites as well as the leader satellite. The coupling effect between the attitude and translational dynamics of the satellites is assumed to be weak and is ignored. Importantly, the follower satellites have information about the control forces employed in the leader.

Hill's equations [2] are considered and consist of relative dynamics in the radial, tangential and out-of-plane direction. However, the out-of-plane dynamics is decoupled, and only the radial and tangential $(x-y)$ plane dynamics, which are coupled, is addressed in this paper. The Hill equations representing the dynamics in the $(x-y)$ plane can be written as:

$$
\begin{aligned}
\ddot{x}_{i}-2 \dot{y}_{i}-3 x_{i} & =u_{x i}+d_{x i} \\
\ddot{y}_{i}+2 \dot{x}_{i} & =u_{y i}+d_{y i}
\end{aligned}
$$

where $x_{i}$ and $y_{i}$ represent the displacements in the radial and tangential directions respectively with respect to the leader satellite, which performs a circular orbit at an angular speed of $\omega_{n}$. Note that (1) -(2) have been normalised with respect to time, and have no visible dependency on $\omega_{n}$ as written [2], [6], [7]. The control signals $u_{x i}$ and $u_{y i}$ are the net specific control forces, in the radial and tangential plane respectively, acting on the $i^{t h}$ follower satellite. These are relative with respect to the leader and can be written as

$$
\begin{aligned}
& u_{x i}=u_{x i}^{f}-u_{x i}^{l} \\
& u_{y i}=u_{y i}^{f}-u_{y i}^{l}
\end{aligned}
$$

where the superscripts $f$ and $l$ indicate the follower and leader respectively, and so for example, $u_{x i}^{f}$ is the control signal applied to the $i^{\text {th }}$ follower satellite in the radial direction. The terms $d_{x i}$ and $d_{y i}$ are the net specific disturbances experienced by the follower satellites due to atmospheric drag, tesseral resonance, $J_{2}$ effects from the solar radiation pressure, and the oblateness of the Earth.

The dynamics of the $i^{\text {th }}$ satellite in the radial and tangential $(x-y)$ plane (1) - (2) can be rewritten conveniently in state space form as in [8], [12], [20]:

$$
\begin{aligned}
\dot{X}_{i} & =A X_{i}+B U_{i}+B d_{i} \\
Z_{i j} & =C\left(X_{i}-X_{j}\right), \quad j \in \mathcal{J}_{i}
\end{aligned}
$$

for $i=1, \ldots, N$ where $X_{i}=\operatorname{Col}\left(x_{1 i}, x_{2 i}, x_{3 i}, x_{4 i}\right):=$ $\operatorname{Col}\left(x_{i}, \dot{x}_{i}, y_{i}, \dot{y}_{i}\right)$ represents the satellite relative states. The coordinates $x_{1 i}:=x_{i}$ and $x_{3 i}:=y_{i}$ describe the position of the $i^{\text {th }}$ follower satellite relative to the leader satellite. In (5)-(6), $U_{i}=\operatorname{Col}\left(u_{x i}, u_{y i}\right)$ represents the control input vector - the net specific control forces acting on the $i^{\text {th }}$ follower satellite and $d_{i}=\operatorname{Col}\left(d_{x i}, d_{y i}\right)$ represents the net specific disturbances respectively. The constant matrices in (5) - (6) are given by

$A=\left[\begin{array}{cccc}0 & 1 & 0 & 0 \\ 3 & 0 & 0 & 2 \\ 0 & 0 & 0 & 1 \\ 0 & -2 & 0 & 0\end{array}\right], B=\left[\begin{array}{ll}0 & 0 \\ 1 & 0 \\ 0 & 0 \\ 0 & 1\end{array}\right], C=\left[\begin{array}{llll}1 & 0 & 0 & 0 \\ 0 & 0 & 1 & 0\end{array}\right]$

The nonempty set $\mathcal{J}_{i} \subset\{1, \ldots, N\} /\{i\}$ represents the indices of the other dynamical systems, for which the $i^{\text {th }}$ dynamical system has information. Here, an assumption is made that each dynamical system has information about at least one other dynamical system. Combining all the relative information among the dynamical systems, at the $i^{\text {th }}$ node level, equation (6) can be written as

$$
Z_{i}=\sum_{j \in \mathcal{J}_{i}} C\left(X_{i}-X_{j}\right)
$$

This notation is consistent with that reported in [12].

Collectively at a network level, the dynamics in (5) - (6) can be written using the Kronecker product notation as

$$
\begin{aligned}
\dot{X} & =\left(I_{N} \otimes A\right) X+\left(I_{N} \otimes B\right)(U+d) \\
Z & =(\mathcal{L} \otimes C) X
\end{aligned}
$$

where $X=\operatorname{Col}\left(X_{1}, \ldots, X_{N_{+}}\right)$represents the concatenated column vector of the $N$ follower satellite states, $U=\operatorname{Col}\left(U_{1}, \ldots, U_{N}\right)$ represents the concatenated control input vector, and $d=\operatorname{Col}\left(d_{1}, \ldots, d_{N}\right)$ represents the net disturbance vector. The Laplacian of the graph $\mathcal{G}$, written as $\mathcal{L} \in \mathbb{R}^{N \times N}$, represents the relative sensing topology in (7). The Laplacian matrix $\mathcal{L}$ is defined as follows:

$$
\begin{aligned}
\mathcal{L}_{i i} & =\left|\mathcal{J}_{i}\right| \\
\mathcal{L}_{i j} & = \begin{cases}-1, & j \in \mathcal{J}_{i} \\
0 & j \notin \mathcal{J}_{i}\end{cases}
\end{aligned}
$$

where $\left|\mathcal{J}_{i}\right|$ is the cardinality of the set $\mathcal{J}_{i}$ and represents the degree of the $i^{t h}$ node. The smallest eigenvalue of $\mathcal{L}$ is zero and the corresponding eigenvector is given by $\mathbf{1}$, a column vector composed entirely of ones. The matrix $\mathcal{L}$ is always rank deficient, symmetric and positive semi-definite in the case of undirected graphs. The Laplacian of a graph with bidirectional communication has identical properties to that of an undirected graph.

\section{A. Problem Definition}

The main objective of this paper is to determine the $u_{x i}$ and $u_{y i}$ control forces required for each satellite to maintain the formation flight. Since it is assumed that the information from the leader satellite about the $u_{x i}^{l}$ and $u_{y i}^{l}$ is broadcast to all the follower satellites, from equation (3) - (4), $u_{x i}^{f}$ and $u_{y i}^{f}$ can be determined provided $U_{i}$ is calculated by the $i^{\text {th }}$ satellite.

A distributed control law $U_{i}$ for $i=1, \ldots, N$ is proposed, based on a polytopic representation of the spatially distributed network, to attain the formation of the dynamics in (5)-(6). The control law $U_{i}$ is assumed to be a function of estimates of $X_{i}$ and measurements $Z_{i}$. Since only relative positions $x_{1 i}$ and $x_{3 i}$ are available a novel nonlinear observer which has some roots in a second order sliding mode control methodology [24], [25] will be employed to reconstruct estimates of $X_{i}$ in finite time. Note that only relative position measurements are available at each node level. The proposed nonlinear observer can be used to reconstruct the entire relative state information in finite time. The distributed control law makes use of the reconstructed (estimated) internal state measurements, plus the relative output (relative position alone) measurements.

An architecture consisting of a novel nonlinear observer and a distributed output feedback controller is proposed for obtaining the formation of the satellites which is described in sequel.

\section{SteP 1: Nonlinear OBSERVER DeSIGN}

The nonlinear observer which is proposed in this paper has its roots in the second order super twisting observer proposed in [24], [25]. It will be designed to simultaneously estimate robustly the entire states and the unknown disturbances, $d_{i}=\operatorname{Col}\left(d_{x i}, d_{y i}\right)$, from the measured relative 
position outputs $\left(x_{1 i}, x_{3 i}\right)$ in each follower satellite. The observer will be driven by the inputs and measured position outputs of the follower satellite local to the $i^{\text {th }}$ follower satellite.

Let the state estimate of the $i^{\text {th }}$ follower satellite be $\tilde{X}_{i}:=\operatorname{Col}\left(\tilde{x}_{1 i}, \tilde{x}_{2 i}, \tilde{x}_{3 i}, \tilde{x}_{4 i}\right)$. Consider the nonlinear observer dynamical system described by

$$
\begin{aligned}
& \dot{\tilde{x}}_{1 i}=\tilde{x}_{2 i}-k_{1}\left|e_{1 i}\right|^{\frac{1}{2}} \operatorname{sgn}\left(e_{1 i}\right) \\
& \dot{\tilde{x}}_{2 i}=3 \tilde{x}_{1 i}+2 \tilde{x}_{4 i}-k_{3} \operatorname{sgn}\left(e_{1 i}\right)-k_{2}\left|e_{3 i}\right|^{\frac{1}{2}} \operatorname{sgn}\left(e_{3 i}\right)+u_{x i} \\
& \dot{\tilde{x}}_{3 i}=\tilde{x}_{4 i}-k_{2}\left|e_{3 i}\right|^{\frac{1}{2}} \operatorname{sgn}\left(e_{3 i}\right) \\
& \dot{\tilde{x}}_{4 i}=-2 \tilde{x}_{2 i}-k_{4} \operatorname{sgn}\left(e_{3 i}\right)+k_{1}\left|e_{1 i}\right|^{\frac{1}{2}} \operatorname{sgn}\left(e_{1 i}\right)+u_{y i}
\end{aligned}
$$

where: $e_{i}=\tilde{X}_{i}-X_{i}$, such that $e_{i}=\operatorname{Col}\left(e_{1 i}, e_{2 i}, e_{3 i}, e_{4 i}\right)$. The $k_{i} \in \mathbb{R}^{+}, i=1, \ldots, 4$ represent the positive design scalar gains to be determined. This will be discussed in the sequel. The global error estimate at the network level is given by $e=\operatorname{Col}\left(e_{1}, \ldots, e_{N}\right)$.

Remark 1: When compared to the classical supertwisting observer proposed in [25], additional significant cross coupling terms $-k_{2}\left|e_{3 i}\right|^{\frac{1}{2}} \operatorname{sgn}\left(e_{3 i}\right)$ and $+k_{1}\left|e_{1 i}\right|^{\frac{1}{2}} \operatorname{sgn}\left(e_{1 i}\right)$ are present in (13) and (15). Furthermore the skew symmetry in the coupling of the states in the satellite dynamics is exploited in proposing the new nonlinear observer. The proposed nonlinear observer will be analysed making use of the class of Lyapunov function originally proposed in [24]. A novelty of the proposed nonlinear observer above is the exploitation of the skew symmetric cross coupling in the satellite plant.

The error in the state estimate of the $i^{t h}$ follower satellite, $e_{i}$, is given by

$$
\begin{aligned}
& \dot{e}_{1 i}=-k_{1}\left|e_{1 i}\right|^{\frac{1}{2}} \operatorname{sgn}\left(e_{1 i}\right)+e_{2 i} \\
& \dot{e}_{2 i}=3 e_{1 i}+2 e_{4 i}-k_{3} \operatorname{sgn}\left(e_{1 i}\right)-k_{2}\left|e_{3 i}\right|^{\frac{1}{2}} \operatorname{sgn}\left(e_{3 i}\right)-d_{x i} \\
& \dot{e}_{3 i}=-k_{2}\left|e_{3 i}\right|^{\frac{1}{2}} \operatorname{sgn}\left(e_{3 i}\right)+e_{4 i} \\
& \dot{e}_{4 i}=-2 e_{2 i}-k_{4} \operatorname{sgn}\left(e_{3 i}\right)+k_{1}\left|e_{1}\right|^{\frac{1}{2}} \operatorname{sgn}\left(e_{1 i}\right)-d_{y i}
\end{aligned}
$$

where $i=1, \ldots, N$. Note that $e_{1 i}$ and $e_{3 i}$ are available and represent the difference between the estimated and the measured relative $x$ and $y$ positions of the $i^{t h}$ satellite. However, the terms $d_{x i}$ and $d_{y i}$ are unknown.

Assumption 3.1: It is assumed that the unknown disturbance terms $d_{x i}$ and $d_{y i}$ in the error dynamics satisfy apriori known upper bounds. Specifically suppose $\left|d_{x i}\right| \leq \delta_{1}$ and $\left|d_{y i}\right| \leq \delta_{2}$ for known constants $\delta_{1}, \delta_{2} \geq 0$. This assumption is similar to the one made in [6], [7].

Consider a candidate function $V\left(e_{i}\right)$ for the error dynamics system in (16) - (19), which is inspired by the one in [24] given by:

$$
\begin{aligned}
V\left(e_{i}\right) & =2 k_{3}\left|e_{1 i}\right|+\frac{1}{2} e_{2 i}^{2}+\frac{1}{2}\left(k_{1}\left|e_{1 i}\right|^{\frac{1}{2}} \operatorname{sgn}\left(e_{1 i}\right)-e_{2 i}\right)^{2} \\
& +2 k_{4}\left|e_{3 i}\right|+\frac{1}{2} e_{4 i}^{2}+\frac{1}{2}\left(k_{2}\left|e_{3 i}\right|^{\frac{1}{2}} \operatorname{sgn}\left(e_{3 i}\right)-e_{4 i}\right)^{2}
\end{aligned}
$$

Note $V\left(e_{i}\right)$ is a continuous positive definite function for all $e_{i}$, but is not differentiable at $\left\{e_{i} \mid e_{1 i}=0, e_{3 i}=0\right\}$. Following the arguments in Remark 1 of [24], Lyapunov methods can still be applied to those points where $V\left(e_{i}\right)$ is differentiable, i.e. for all $\left\{e_{i}=\operatorname{Col}\left(e_{1 i}, e_{2 i}, e_{3 i}, e_{4 i}\right) \mid e_{1 i} \neq\right.$ $\left.0, e_{3 i} \neq 0\right\}$. In the sequel, it will be shown that $V\left(e_{i}\right)$ is indeed a Lyapunov function for system (16) - (19).
For simplicity, the proposed candidate Lyapunov function can be written as a quadratic form $V\left(\xi_{i}\right)=\xi_{i}^{\mathrm{T}} P \xi_{i}$ where $\xi_{i}:=\operatorname{Col}\left(\xi_{i 1}, \xi_{i 2}\right)$ and $\xi_{i 1}:=\operatorname{Col}\left(\left|e_{1 i}\right|^{\frac{1}{2}} \operatorname{sgn}\left(e_{1 i}\right), e_{2 i}\right)$ and $\xi_{i 2}:=\operatorname{Col}\left(\left|e_{3 i}\right|^{\frac{1}{2}} \operatorname{sgn}\left(e_{3 i}\right), e_{4 i}\right)$. The block diagonal Lyapunov matrix

$$
P=\left[\begin{array}{cc}
P_{1} & \mathbf{0}_{2 \times 2} \\
\mathbf{0}_{2 \times 2} & P_{2}
\end{array}\right]
$$

where

$$
P_{1}=\frac{1}{2}\left[\begin{array}{cc}
4 k_{3}+k_{1}^{2} & -k_{1} \\
-k_{1} & 2
\end{array}\right], \quad P_{2}=\frac{1}{2}\left[\begin{array}{cc}
4 k_{4}+k_{2}^{2} & -k_{2} \\
-k_{2} & 2
\end{array}\right]
$$

and radially unbounded if $k_{3}>0$ and $k_{4}>0$. It can be shown that the time derivative of $V\left(\xi_{i}\right)$ along the trajectories of the system (16) - (19) is given by

$$
\dot{V}\left(\xi_{i}\right)=-\frac{1}{\left|e_{1 i}\right|^{\frac{1}{2}}} \xi_{i 1}^{\mathrm{T}} Q_{1} \xi_{i 1}-\frac{1}{\left|e_{3 i}\right|^{\frac{1}{2}}} \xi_{i 2}^{\mathrm{T}} Q_{2} \xi_{i 2}+\xi_{i}^{\mathrm{T}} Q_{3} d_{i}
$$

where

$$
Q_{1}=\frac{k_{1}}{2}\left[\begin{array}{cc}
2 k_{3}+k_{1}^{2} & -k_{1} \\
-k_{1} & 1
\end{array}\right], \quad Q_{2}=\frac{k_{2}}{2}\left[\begin{array}{cc}
2 k_{4}+k_{2}^{2} & -k_{2} \\
-k_{2} & 1
\end{array}\right]
$$

and

$$
Q_{3}=\left[\begin{array}{cc}
-k_{1} & 0 \\
2 & 0 \\
0 & -k_{4} \\
0 & 2
\end{array}\right]
$$

Note that significant algebraic manipulation is necessary to achieve the structure in (22) because although $V\left(\xi_{i}\right)$ and $\dot{V}\left(\xi_{i}\right)$ present a decoupled block structure as given in (20) and (22), the differential equations in (16)-(19) are coupled. In achieving (22) the skew symmetry of the satellite plant and the additional coupling terms mentioned in Remark 3 have been exploited.

It is clear $V\left(\xi_{i}\right)$ is negative definite if $Q_{1}>0$ and $Q_{2}>0$ for all $\xi_{1 i} \neq 0$ and $\xi_{3 i} \neq 0$. Note that from (22), this condition is true for all choice of $k_{i}>0$ for $i=1, \ldots 4$ when the terms $d_{x i}$ and $d_{y i}$ equal to zero. Hence, for $k_{i}>$ $0, i=1, \ldots 4$ and $d_{x i}=d_{y i}=0$, all the trajectories of system (16) - (19) converge in finite time (shown in sequel) to the origin $e_{i}=0$.

From assumption 1, the upper bounds on the unknown disturbance terms $d_{x i}$ and $d_{y i}$ are known. In the presence of these bounded unknown disturbance terms, it can be shown using arguments similar to those in [24] that

$$
\dot{V}\left(\xi_{i}\right) \leq-\frac{1}{\left|e_{1 i}\right|^{\frac{1}{2}}} \xi_{i 1}^{\mathrm{T}} \tilde{Q}_{1} \xi_{i 1}-\frac{1}{\left|e_{3 i}\right|^{\frac{1}{2}}} \xi_{i 2}^{\mathrm{T}} \tilde{Q}_{2} \xi_{i 2}
$$

where

$$
\tilde{Q}_{1}=\frac{k_{1}}{2}\left[\begin{array}{cc}
2 k_{3}+k_{1}^{2}-2 \delta_{1} & -k_{1}-2 \frac{\delta_{1}}{k_{1}} \\
-k_{1}-2 \frac{\delta_{1}}{k_{1}} & 1
\end{array}\right]
$$

and

$$
\tilde{Q}_{2}=\frac{k_{2}}{2}\left[\begin{array}{cc}
2 k_{4}+k_{2}^{2}-2 \delta_{2} & -k_{2}-2 \frac{\delta_{2}}{k_{1}} \\
-k_{2}-2 \frac{\delta_{2}}{k_{1}} & 1
\end{array}\right]
$$

Now in this situation $\dot{V}\left(\xi_{i}\right)$ is negative definite if $\tilde{Q}_{1}$ and $\tilde{Q}_{1}$ are positive definite. Provided the scalar positive gains $k_{i}$, for $i=1, \ldots, 4$, satisfy the following conditions

$$
\begin{array}{ll}
k_{1}>0, & k_{3}>3 \delta_{1}+2 \frac{\delta_{1}^{2}}{k_{1}^{2}} \\
k_{2}>0, & k_{4}>3 \delta_{2}+2 \frac{\delta_{2}^{2}}{k_{2}^{2}}
\end{array}
$$


it can be seen that $\tilde{Q}_{1}$ and $\tilde{Q}_{1}$ are positive definite and consequently $\dot{V}\left(\xi_{i}\right)$ is negative definite for all $\xi_{i} \neq 0$ and $t>0$.

\section{A. Finite time convergence to origin $e_{i}=0\left(\xi_{i}=0\right)$}

Exploiting the very specific block diagonal structure of the Lyapunov matrix in (21), rewrite the quadratic Lyapunov function in (20) as

$$
V\left(\xi_{i}\right):=\underbrace{\xi_{i 1}^{\mathrm{T}} P_{1} \xi_{i 1}}_{V_{1}\left(\xi_{i 1}\right)}+\underbrace{\xi_{i 2}^{\mathrm{T}} P_{2} \xi_{i 2}}_{V_{2}\left(\xi_{i 2}\right)}
$$

The functions $V_{1}\left(\xi_{i 1}\right)$ and $V_{2}\left(\xi_{i 2}\right)$ are positive definite with respect to $\xi_{i 1}$ and $\xi_{i 2}$ respectively. From Rayleigh's inequality [27]

$$
\begin{aligned}
& \gamma_{\min }\left(P_{1}\right)\left\|\xi_{i 1}\right\|_{2}^{2} \leq V_{1}\left(\xi_{i 1}\right) \leq \gamma_{\max }\left(P_{1}\right)\left\|\xi_{i 1}\right\|_{2}^{2} \\
& \gamma_{\min }\left(P_{2}\right)\left\|\xi_{i 2}\right\|_{2}^{2} \leq V_{2}\left(\xi_{i 2}\right) \leq \gamma_{\max }\left(P_{2}\right)\left\|\xi_{i 2}\right\|_{2}^{2}
\end{aligned}
$$

where $\gamma_{\min }($.$) and \gamma_{\max }($.$) represent the minimum and$ maximum eigenvalue of the Lyapunov matrix, and $\|\cdot\|_{2}$ represents the Euclidean norm. It can be shown that

$$
\left|e_{1}\right|^{\frac{1}{2}} \leq\left\|\xi_{i 1}\right\|_{2} \leq \frac{V_{1}^{\frac{1}{2}}\left(\xi_{i 1}\right)}{\gamma_{\text {min }}^{\frac{1}{2}}\left(P_{1}\right)}
$$

and

$$
\left|e_{3}\right|^{\frac{1}{2}} \leq\left\|\xi_{i 2}\right\|_{2} \leq \frac{V_{2}^{\frac{1}{2}}\left(\xi_{i 2}\right)}{\gamma_{\min }^{\frac{1}{2}}\left(P_{2}\right)}
$$

then following identical arguments to those in [24], the inequality in (23) can be written as

$\dot{V}\left(\xi_{i}\right) \leq-\frac{1}{\left|e_{1 i}\right|^{\frac{1}{2}}} \gamma_{\min }\left(\tilde{Q}_{1}\right)\left\|\xi_{i 1}\right\|_{2}^{2}-\frac{1}{\left|e_{3 i}\right|^{\frac{1}{2}}} \gamma_{\min }\left(\tilde{Q}_{2}\right)\left\|\xi_{i 2}\right\|_{2}^{2}$

Subsequently using the inequalities (29) and (30), (31) can further be written as

$$
\dot{V}\left(\xi_{i}\right) \leq-\beta_{1} V_{1}^{\frac{1}{2}}\left(\xi_{i 1}\right)-\beta_{2} V_{2}^{\frac{1}{2}}\left(\xi_{i 2}\right)
$$

where $\beta_{1}=\frac{\gamma_{\min }^{\frac{1}{2}}\left(P_{1}\right) \gamma_{\min }\left(\tilde{Q}_{1}\right)}{\gamma_{\max }\left(P_{1}\right)}$ and $\beta_{2}=\frac{\gamma_{\min }^{\frac{1}{2}}\left(P_{2}\right) \gamma_{\min }\left(\tilde{Q}_{2}\right)}{\gamma_{\max }\left(P_{2}\right)}$ and thus it follows that

$$
\dot{V}\left(\xi_{i}\right) \leq-\beta\left(V_{1}^{\frac{1}{2}}\left(\xi_{i 1}\right)+V_{2}^{\frac{1}{2}}\left(\xi_{i 2}\right)\right)
$$

where $\beta=\min \left(\beta_{1}, \beta_{2}\right)$. Since $\left(V_{1}^{\frac{1}{2}}+V_{2}^{\frac{1}{2}}\right)^{2}>V_{1}+V_{2}$, because $V_{1}$ and $V_{2}$ are positive, it can be concluded that $V_{1}^{\frac{1}{2}}+V_{2}^{\frac{1}{2}}>V^{\frac{1}{2}}$. This further implies that

$$
\dot{V}\left(\xi_{i}\right) \leq-\beta V^{\frac{1}{2}}
$$

and hence $V\left(\xi_{i}\right) \equiv 0$ in finite time.

\section{$B$. Reconstruction of states and disturbance}

As argued above, the origin $e_{i}=0$ is attained in finite time. Consequently from (12) - (15), the estimates of the states of the $i^{\text {th }}$ follower satellite $\tilde{X}_{i}:=$ $\operatorname{Col}\left(\tilde{x}_{1 i}, \tilde{x}_{2 i}, \tilde{x}_{3 i}, \tilde{x}_{4 i}\right)$ is available in finite time. Substituting for $e_{i} \equiv 0$ in (17) and (19) yields

$$
\begin{aligned}
& \underbrace{k_{3} \operatorname{sgn}\left(e_{1 i}\right)}_{\nu_{1 i}}-d_{x i}=0 \\
& \underbrace{k_{4} \operatorname{sgn}\left(e_{3 i}\right)}_{\nu_{3 i}}-d_{y i}=0
\end{aligned}
$$

Therefore $\nu_{e q, 1 i}:=d_{x i}$ and $\nu_{e q, 3 i}:=d_{y i}$, where $\nu_{e q, i *}$ denotes the equivalent injection signals [28] necessary to maintain sliding. Thus $d_{x i}$ and $d_{y i}$ can be obtained to good accuracy by low pass filtering of $\nu_{1 i}$ and $\nu_{3 i}$ [28]. The information about the estimates of $d_{x i}$ and $d_{y i}$, defined as $\tilde{d}_{x i}$ and $\tilde{d}_{y i}$ respectively, can be employed in the control law to improve the disturbance rejection properties. The observer in (12)-(15) clearly has roots in super twist observer from [25], that has already been used for FDI in a specific satellite leader/follower configuration [26]. However in this paper, the dynamics of the radial and tangential components are coupled via skew symmetric terms that are explicitly accounted for in the bespoke observer (12)-(15) proposed in this paper.

\section{Step 2: Controller Part Design}

The proposed controller consists of two parts, a local internal state feedback component, (since all the internal states $X_{i}$ are available at each follower satellite in finite time from the proposed nonlinear observer), and a distributed component which depends on the external relative measured positions $\left(Z_{i}\right)$ of the follower satellites. Consider a control law of the form

$$
U_{i}=-K X_{i}-Z_{i}-\tilde{d}_{i}
$$

for $i=1, \ldots, N$, where $K \in \mathbb{R}^{2 \times 4}$ is a state feedback gain and $\tilde{d}_{i}=\operatorname{Col}\left(\tilde{d}_{x i}, \tilde{d}_{y i}\right)$. Using the control law from (37) in a feedback loop, the closed loop dynamics in (5) (assuming for the time being the disturbance $d_{i}=0$ ) is given by

$$
\dot{X}_{i}=(A-B K) X_{i}-B Z_{i}+B\left(d_{i}-\tilde{d}_{i}\right)
$$

for $i=1, \ldots, N$. Ignoring the $\left(d_{i}-\tilde{d}_{i}\right)$ terms, the closed loop dynamics of the overall network can be conveniently written as

$$
\dot{X}=\left(I_{N} \otimes(A-B K)\right) X-(\mathcal{L} \otimes B C) X
$$

where $X=\operatorname{Col}\left(X_{1}, \ldots, X_{N}\right)$ and $\mathcal{L}$ represents the connectivity associated with the relative sensing among the satellites which is represented in terms of an undirected graph (since a bidirectional communication architecture is assumed). The matrix $\mathcal{L}$ is symmetric and hence a spectral decomposition is possible. Consequently $\mathcal{L}$ can be written as:

$$
\mathcal{L}=S \Lambda S^{\mathrm{T}}
$$

where $S$ is an orthogonal matrix constituting of the eigenvectors of $\mathcal{L}$ and $\Lambda$ is a diagonal matrix with the eigenvalues of $\mathcal{L}$ as the diagonal terms, which all are real numbers. The diagonal matrix $\Lambda$ is

$$
\Lambda:=\operatorname{Diag}\left(\lambda_{1}, \ldots, \lambda_{i}, \ldots, \lambda_{N}\right)
$$

with the property

$$
0=\lambda_{1}<\lambda_{2} \leq \ldots \leq \lambda_{i} \leq \ldots \leq \lambda_{N}=\lambda_{\max }
$$

Define a coordinate transformation $X \mapsto \bar{X}:=T X$ where $T$ is defined as

$$
T:=\left(S^{\mathrm{T}} \otimes I_{n}\right)
$$

Because $S$ is an orthogonal matrix obtained from (40), it can be shown by making use of Kronecker product properties that, the transformation in (43) is orthogonal. Applying the coordinate transformation $T: X \mapsto \bar{X}$ in (43), the closed loop dynamics of the overall network is given by

$$
\dot{\bar{X}}=\left(\left(I_{N} \otimes(A-B K)\right)-(\Lambda \otimes B C)\right) \bar{X}
$$


where $\bar{X}=\operatorname{Col}\left(\bar{X}_{1}, \ldots, \bar{X}_{N}\right)$. Since $\Lambda$ is the diagonal matrix in (41), a decoupling is achieved in the new coordinate system, when compared to the closed loop dynamics of the overall network in (39). As a result of the transformation, the dynamics in (44) can be written in the form

$$
\dot{\bar{X}}_{i}=\left(A-B K-\lambda_{i} B C\right) \bar{X}_{i}
$$

for $i=1, \ldots, N$. Since each $\lambda_{i}$ satisfies $0<\lambda_{i}<$ $\lambda_{N}$ the problem of choosing $K$ can be thus viewed as involving stabilizing a polytopic system [29], [30] where a real parameter $\lambda_{i}$ varies in an interval $\left[0, \lambda_{\max }\right]$. Consider the dynamics in (45) as a polytopic system $\mathcal{P}(\lambda)$ where $\lambda$ varies in the interval $\left[0, \lambda_{\max }\right]$ and thus the dynamics in (45) can be treated as a convex combination of $\mathcal{P}_{\text {min }}$ and $\mathcal{P}_{\text {max }}$, the two plants defined at the extremes of the interval. Write $\mathcal{P}(\lambda)$ as

$$
\mathcal{P}(\lambda)=\rho_{1} \mathcal{P}_{\text {min }}+\rho_{2} \mathcal{P}_{\text {max }}
$$

where $\rho_{1}=1-\frac{\lambda}{\lambda_{\max }}$ and $\rho_{2}=\frac{\lambda}{\lambda_{\max }}$ so that $\rho_{1}+\rho_{2}=1$ and hence the plant $P(\lambda)$ is affine in $\lambda$. In equation (45), the plants corresponding to the extreme variations of $\lambda$ are given as:

$$
\begin{aligned}
& \mathcal{P}_{\min }:=A-B K \\
& \mathcal{P}_{\max }:=A-B K+\lambda_{\max } B C
\end{aligned}
$$

Hence, the problem can be posed as one of finding controller gain matrices $K$ and a common Lyapunov matrix $M \in \mathbb{R}^{4 \times 4}$ to stabilise the family of plants defined by $\mathcal{P}(\lambda) \in\left[\mathcal{P}_{\text {min }}, \mathcal{P}_{\text {max }}\right]$.

$$
\begin{aligned}
& (A-B K)^{\mathrm{T}} M+M(A-B K)<-\delta I \\
& \left(A-\lambda_{\max } B C-B K\right)^{\mathrm{T}} M+M\left(A-\lambda_{\max } B C-B K\right)<-\delta I \\
& M>0
\end{aligned}
$$

Since $M$ and $K$ are unknown, the above inequalities are not a system of LMI's in variables $M$ and $K$. However, if new variables are introduced such as $\tilde{M}=M^{-1}, \tilde{K}=K \tilde{M}$, then the inequalities (49)-(51) read as

$$
\begin{aligned}
& A \tilde{M}+\tilde{M} A^{\mathrm{T}}+B \tilde{K}+\tilde{K}^{\mathrm{T}} B<-\delta I \\
& \left(A-\lambda_{\max } B C\right) \tilde{M}+\tilde{M}\left(A-\lambda_{\max } B C\right)^{\mathrm{T}}+B \tilde{K}+\tilde{K}^{\mathrm{T}} B<-\delta I( \\
& \tilde{M}>0
\end{aligned}
$$

The inequalities (52) - (54) are a system of LMI's in the decision variables $\tilde{M}$ and $\tilde{K}$. The problem has thus a solution $K=\tilde{K} \tilde{M}^{-1}$, provided the system of LMI's are feasible [30]. Additional LMIs can be incorporated to penalize control effort and to tune the response of the closed loop system [29], [30].

The disturbances $d_{i}$ terms present in (5), can be estimated in finite time by the proposed nonlinear observer in (12) (19). The estimates can then be used to nullify the effect of the disturbances in the closed loop, to ensure $B\left(d_{i}-\tilde{d}_{i}\right) \approx 0$ in (38) to obtain good disturbance rejection.

\section{RESUlTS}

A satellite cluster consisting of one leader and four follower satellites is considered to demonstrate the proposed framework. The leader satellite is assumed to be following a circular orbit. The follower satellites are required to take up positions on the four corners of a square centered on the leader.

To satisfy assumption 3.1, upper bounds on the unknown disturbances are assumed as $\delta_{1}=1$ and $\delta_{2}=1$. The gains $k_{i}$ for $i=1, \ldots, 4$ need to be selected satisfying the conditions (24)-(25). In the following simulations the gains of the nonlinear observer associated with each satellite are given by $k_{1}=1, k_{2}=1, k_{3}=10$ and $k_{4}=10$. These values satisfy the stability conditions in (24)-(25). In terms of the control law, a feasible solution to the LMIs in (52)(54) has been found for $\delta=1$ whilst minimising the norm of the controller gain. In fig. 1, the evolution of the state estimation errors are shown. Clearly the states $X_{1}$ are all reconstructed perfectly in finite time. Figure 2 shows the

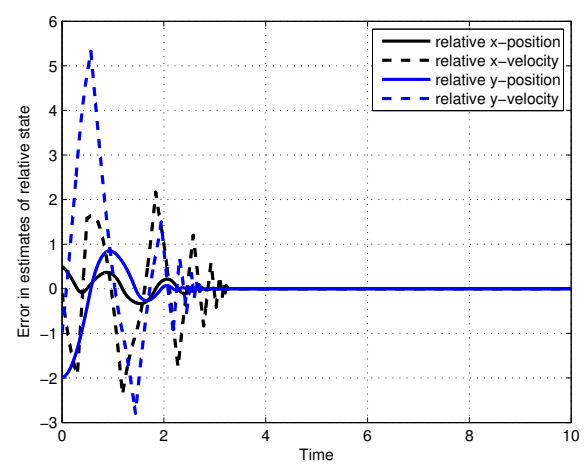

Fig. 1. Error in estimates of relative states

four follower satellites taking up the required formation (depicted in a radial-tangential plane with respect to the leader axis) centered on the leader.

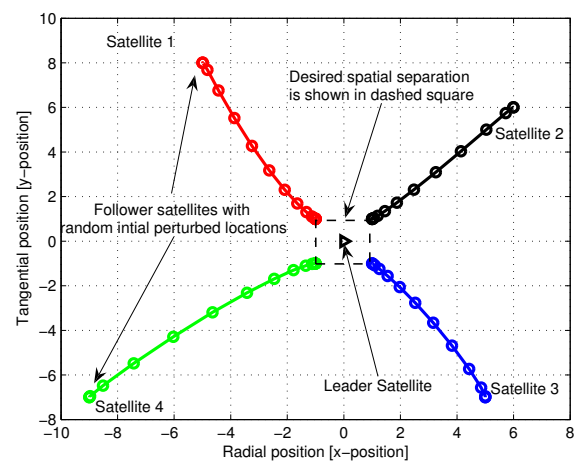

Fig. 2. Formation of 4 Satellites viewed from the leader frame

In the simulation shown above, no disturbance terms are present, i.e, $d_{i}=0$ for $i=1, \ldots, 4$. For demonstration purposes in the sequel sinusoidal terms are added to impact on Satellite 1. The disturbances are given by $d_{i x}=a_{0} \sin \left(\omega_{0} t\right)$ and $d_{i y}=a_{1} \sin \left(\omega_{1} t\right)$ where $a_{0}=$ $0.75, a_{1}=0.5$ and $\omega_{0}=\omega_{1}=5$. Figure 3 shows the plots of the equivalent signals $\nu_{e q, 1}$ and $\nu_{e q, 3}$ satisfying (35) and (36). Clearly the injection terms track the disturbance terms $d_{1 x}$ and $d_{1 y}$ in finite time. Figure 4 shows the effect of the disturbance terms on the closed loop performance where $\tilde{d}_{1 x}=\nu_{e q, 1}$ and $\tilde{d}_{1 y}=\nu_{e q, 3}$ are used in the controller to provide further robustness and disturbance rejection.

\section{CONCLUSION}

Formation control of a cluster of satellites has been addressed using a combination of a nonlinear observer and a distributed controller. While designing the nonlinear 

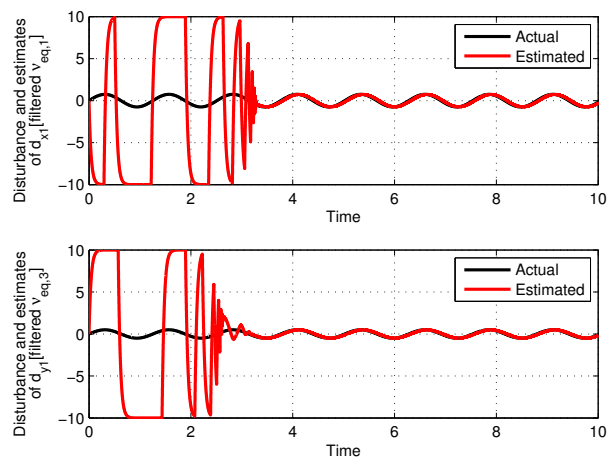

Fig. 3. Estimates in disturbances

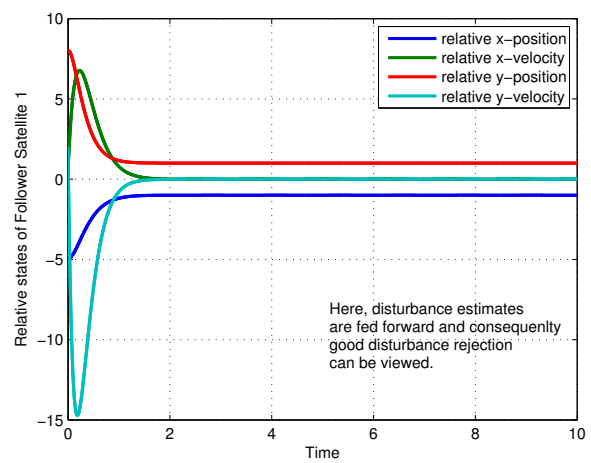

Fig. 4. Relative states when estimates of disturbances are fedforward

observer, the skew symmetry of the satellite dynamics has been exploited. The proposed nonlinear observer builds on the principles of the super-twist sliding mode observer. Estimation of the entire states and unknown bounded disturbances in finite time is demonstrated using an elegant global Lyapunov analysis.

A distributed controller is realized that makes use of the state estimates and the relative position output information, which depends on the underlying communication topology. The novelty in the synthesis of the controller is mainly in the treatment of the underlying graph topology, the interaction among the satellites in terms of relative sensing, and the synthesis of the controller gains using a simple polytopic representation that depends on the graph Laplacian eigenvalues. A cluster of satellites consisting of a leader and four followers in a circular orbit is considered, all represented using Hill's equations. The efficacy of the proposed nonlinear observer is demonstrated explicitly.

\section{REFERENCES}

[1] V. Kapila, A. G. Sparks, J. M. Buffington, and Q. Yan, "Spacecraft Formation Flying: Dynamics and Control," AIAA Journal of Guidance Control and Dynamics, vol. 23, no. 3, pp 561-564, 2000.

[2] H. H. Yeh and A. Sparks, "Geometry and Control of Satellite Formations," In Proceedings of the ACC, Chicago, Illinois, 2000.

[3] D. P. Scharf, F. Y. Hadaegh, and S. R. Ploen, "A Survey of Spacecraft Formation Flying Guidance and Control (Part 1): Guidance," Proceedings of the ACC, New Jersey, pp. 1733-1739, 2003.

[4] D. P. Scharf, F. Y. Hadaegh, and S. R. Ploen, "A Survey of Spacecraft Formation Flying Guidance and Control (Part 2): Control," Proceedings of the ACC, New Jersey, pp. 1733-1739, 2003.

[5] W. H. Clohessy, R. H. Wiltshire, "Terminal Guidance System for Satellite Rendezvous," Journal of Aerospace Science, vol. 27, pp 653$658,1960$.
[6] H. H. Yeh, E. Nelson and A. Sparks, "Nonlinear Tracking Control for Satellite Formations," AIAA Journal of Guidance Control and Dynamics, vol. 25, no. 2, pp 376-386, 2002.

[7] T. Massey and Y. Shtessel, "Continuous Traditional and High Order Sliding Modes for Satelite Formation Control," AIAA Journal of Guidance Control and Dynamics, vol. 28, no. 4, pp 826-831, 2005.

[8] P. Massioni and M. Verhaegen, "Distributed Control for Identical Dynamically Coupled Systems: A Decomposition Approach,'IEEE Trans. Autom. Control, vol. 54, pp. 124-135, 2009.

[9] R. S. Smith and F. Y. Hadaegh, "Control of Deep Space Formation Flying Spacecraft; Relative Sensing and Switched Information," AIAA Journal of Guidance Control and Dynamics, vol. 28, no. 1, pp 106-114, 2005 .

[10] S. J. Chung, U. Ahsun and J. J. E. Slotine, "Application of Synchronisation to Formation Flying Spacecraft: Lagrangian Approach," AIAA Journal of Guidance Control and Dynamics, vol. 32, no. 2, 2009.

[11] W. Ren and R. W. Beard, Distributed consensus in multi-vehicle cooperative control, Springer, Lecture Notes in Communication and Control Engineering Series, 2007.

[12] J. A. Fax and R. M. Murray, "Information flow and cooperative control of vehicle formations," IEEE Trans. Autom. Control, vol. 49, pp. 1465-1476, 2004

[13] R. Olfati-Saber and R.M. Murray, "Consensus protocols for networks of dynamic agents," In Proc. of Amer. Control Conf., pp. 951-956, 2003.

[14] M. Mesbahi and F. Y. Hadaegh, "Formation Flying of Multiple Spacecrafts via Gaphs, Matrix Inequalities, and Switching," AIAA Journal of Guidance Control and Dynamics, vol. 24, no. 2, pp 369377, 2001

[15] J. Lawton, R. W. Beard and F. Y. Hadaegh, "Elementary Attitude Formation Maneuvers via Leader-Following and Behaviour-Based Control," AIAA paper 2000-4442, August, 2000.

[16] R. Pongvthithum, S. M. Veres, S. B. Gabriel and E. Rogers, "Universal Adaptive Control of Satellite Formation Flying," International Journal of Control, vol. 78, no. 1, pp 45-52, 2005.

[17] H. Wong, H. Pan, M.S. de Queiroz and V. Kapila, "Adaptive Learning Control for Spacecraft Formation Flying," In Proc. of IEEE CDC, Orlando, FL, USA, pp. 10891094, 2001.

[18] P. O. Arambel, C. Rago, and R. K. Mehra, "Covariance Interection Algorithm for Distributed Spacecraft State Estimation," In Proceedings of ACC, Philadelphia, 2001.

[19] F. Y. Hadaegh, B. H. Kang, and D. P. Scharf, "Rule-Based Estimation and Control of Formation Flying Spacecraft," IEEE Conference Fuzzy Systems, Philadelphia, 2001.

[20] M. Mesbahi and M. Egerstedt, Graph Theoretic Methods in Multiagent Networks", Princeton Series in Applied Mathematics, 2010.

[21] M. Tillerson, L. Berger and J. P. How, "Distributed Coordination and Control of Formation Flying," In Proc. of ACC, Philadelphia, pp. 1740-1745, 2003.

[22] P. Ferguson and J. P. How, "Decentralized Estimation Algorithms for Formation Flying Spacecrafts," AIAA Paper 2003-5442, 2003.

[23] R. S. Vadali, S.S. Vaddi, K. Naik, and K. T. Alfred, "Control of Satellite Formations," AIAA Paper 2001-4028, 2001.

[24] J. A. Moreno and M. Osorio, "A Lyapunov Approach to Second Order Sliding Mode Controllers and Observers," In Proc. of 47th IEEE CDC, Cancun, 2008.

[25] J. Davilla, L. Fridman, and A. Levant, "Second-Order Sliding Modes Observer for Mechanical Systems,"IEEE Trans. Autom. Control, vol. 50, no. 11, pp. 1785-1789, 2005.

[26] C. Edwards, L. Fridman, and M. W. Thein, "Fault Reconstruction in a Leader/Follower Spacecraft System Using Higher Order Sliding Mode Observers", In Proc. of ACC, NY, 2007.

[27] H. Khalil, Nonlinear systems, Prentice Hall, $3^{\text {rd }}$ edition, New York, 2002.

[28] V. I. Utkin, Sliding Modes and Their Application in Variable Structure Systems, 1978.

[29] S. Boyd, L. El Ghaoui, E. Feron, and V. Balakrishnan, Linear Matrix Inequalities in system and control theory, SIAM, 1994.

[30] P. Gahinet, A. Nemirovski, A.J. Laub, and M.I. Chilali, LMI control tool box, The MathWorks Inc., 1995 